\title{
The current role of laparoscopic IPOM repair in abdominal wall reconstruction
}

\author{
A. Sharma ${ }^{1} \cdot$ D. Berger ${ }^{2}$
}

Received: 8 June 2018 / Accepted: 25 August 2018 / Published online: 30 August 2018

○) Springer-Verlag France SAS, part of Springer Nature 2018

In the evolution of hernia repair, we have learnt that maximally invasive surgical interventions for hernia repair such as long incisions, extensive tissue mobilization and creation of large myofascial flaps lead to significant morbidity $[1,2]$. Dissection of large tissue planes is associated with morbidity sequelae like wound infection, tissue necrosis, seromas, and hematomas [3]. Long fascial suture lines are potential causes of morbidity [dehiscence, internal hernia (bowel herniation following dehiscence of posterior rectus sheath suture line in eTEP repairs), bleeding, hematoma].

Since the first description by LeBlanc and Booth [4], laparoscopic ventral hernia repair (LVHR) with intraperitoneal mesh placement (IPOM) is now an established surgical procedure for primary and incisional abdominal wall hernias. The intraperitoneal site of mesh placement with the possible sequelae (visceral adhesions, bowel obstruction, fistulization, mesh migration) and bridging repair with prosthesis were concerns raised by some surgeons against the procedure. This led to the quest for an alternative site of mesh placement for hernia repair with minimal access approach. The preperitoneal site for placement of mesh was described (TAPP, transabdominal preperitoneal approach [5] and TAPE, transabdominal partially extraperitoneal approach [6] for peripheral abdominal wall hernias). An endolaparoscopic retromuscular mesh repair for midline hernias was devised (RR approach, eTEP, eRives stoppa). Lateral extensions of the retromuscular (retrorectus) plane were developed after division of transversus abdominis muscle (TAR) for extraperitoneal placement of large meshes extending to paravertebral spaces [7].

A. Sharma

anil.sharma@maxhealthcare.com; asharma736@yahoo.in

1 Max Institute of Minimal Access, Metabolic and Bariatric Surgery, Max Healthcare, Saket, New Delhi, India

2 Privatklinik Lindberg, Schickstrasse 11, Winterthur, Switzerland
Endolaparoscopic retrorectus mesh repair for midline hernias is performed with minimal access, but is surgically maximally invasive. It involves extensive mobilization of large myofascial flaps upto semilunar lines laterally to create requisite retromuscular space for hernia repair and mesh placement. The surgical procedure requires division of the entire linea alba in the midline to create the large retrorectus space required for placement of mesh. With this approach, essentially, repair of a midline hernia necessitates complete division followed by suture of an otherwise normal linea alba ("to fix a leaking tap why change the entire plumbing?"). The normal linea alba is akin to a natural dynamic lattice of criss cross fibres that cross the midline and reinforce the contralateral sheath [8]. A divided and sutured normal linea alba (with mesh reinforcement) is a poor substitute. Additionally, there is potential for damage to important neurovascular perforators laterally near semilunar lines. The learning of these new endoscopic techniques is not easy and the use of robotics to enhance suturing capacities is being suggested.

Component separation techniques have been introduced to facilitate medialisation of the abdominal wall and a tension free reapproximation of abdominal wall constituents for primary closure [9]. ["Steal from Joe (lateral compartment") and provide to "John (medial compartment)"]. With increasing acceptance and performance of different component separation techniques, also done endolaparoscopically, the point of anatomic reconstruction gained increasing significance. These functional aspects are well studied after repair of major abdominal defect with a fascial width of $>10 \mathrm{~cm}$. A significant improvement of function and quality of life by anatomical reconstruction could be shown [10]. As such these are extensive reconstructive procedures of the abdominal wall with attendant morbidity and mortality [11]. It follows that due diligence and caution is mandatory to ensure that these procedures are performed for the right indications. The TA muscle with attachments to the thoracic cage spine and pelvis has been previously described as the 
corset of the abdominal wall. Division of TA muscle has been reported to lead to hypotonia and bulging of the lateral abdominal wall. Only very preliminary observations suggest improvement in core abdominal wall functionality on dynamometry after TAR reconstruction [12]. However, the potential deleterious effect of division and separation of TA muscle on the abdominal wall and respiratory function need to be observed and evaluated in the longer term. The use of multiple large sheets of mesh $(30 \times 30 \mathrm{~cm})$ as reinforcement for the entire abdominal wall raises several concerns. The abdominal cavity has been designed to be flexible and distensible and large sheets of mesh encasing the abdominal wall including the flanks are expected to reduce mobility, and therefore, function. Primarily well-functioning parts of the abdominal wall are either destroyed or at least fixed by these large meshes and the induced scar. Mesh contamination and infection in such a scenario is expected to lead to great morbidity.

In the early days laparoscopic adhesiolysis proved to be the most difficult and hazardous part of the surgical procedure. However, recent meta-analysis do not reveal any disadvantage of laparoscopic repair with regard to unrecognized enterotomies [13]. The intraperitoneal location of foreign material was believed to be associated with adhesions leading to obstruction and enteric fistulization. Major cohort studies at the turn of the century could largely exclude these major sequelae. However, some surgeons experienced severe complications attributed to the technique and the mesh material. One of the authors groups (AS) has recently reported on adhesive characteristics and sequelae during relaparoscopy in patients with previously implanted intraperitoneal meshes [14]. It was reported that intraperitoneal mesh placement is associated with a higher total adhesion score that may increase risk during subsequent laparoscopic surgery. Otherwise adhesion-related complications have been described only anecdotically in terms of case reports. Major series based on well-controlled registry data do exist and rule out short and medium term complications after laparoscopic IPOM repair [15]. The Danish Hernia Database shows that there is a relevant rate of revisional surgery after incisional hernia repair over time due to mesh-related complications [16] for both open and laparoscopic approaches. After 5 years of follow-up the cumulative incidence of meshrelated complications is $5.6 \%$ for open and $3.7 \%$ for laparoscopic repairs. Current literature reveals robust data ruling out any inferiority of laparoscopic hernia repair in terms of mesh-related complications caused by the intraperitoneal placement. Nevertheless a greater understanding and awareness of post-operative adhesive sequelae may possibly lead to safer meshes, better fixation and more refined surgical techniques for open and laparoscopic approaches in future. The use of well-controlled registries is important to identify advantages as well as complications of different procedures.
The indications for laparoscopic IPOM repair are more clearly defined as the procedure has evolved over the decades. The low infection rate make it suitable for use in patients at high risk for infection (diabetes, obesity, immune compromise). It is indicated in patients with recurrent hernias after open repair, in patients with undisturbed abdominal wall function with swiss cheese defects, small fascial defects with large primary incisions (to cover the entire scar) lateral (L1-L3) hernia defects.

Fascial closure has been recommended by some surgeons [17] to prevent bulging of abdominal wall and seromas after bridging repair in laparoscopic IPOM repair [18]. Laparoscopic closure of the fascial defect is technically feasible, yet evidence of any superiority over standard procedure is lacking [19]. Patients with large hernial defects (especially active patients with impairment of abdominal wall function) are unsuitable for laparoscopic IPOM repair [20].

The optimal way forward in surgical science will be driven as much with new innovation as with lessons learnt in the past. Hernia is a benign, non-life threatening condition. However, recent trends in evolution of hernia repair seem to advocate more elaborate and more aggressive surgical interventions on the abdominal wall. The endolaparoscopic retrorectus repair has the advantages of retro muscular (extraperitoneal) mesh placement, potential cost savings on mesh and less fixation of mesh. However, the repair may also lead to unacceptable collateral damage (linea alba) and increased morbidity (large tissue planes, long suture lines, injury to neurovascular perforators). The component separation techniques provide a satisfactory, feasible and reproducible surgical alternative for large, difficult and complex abdominal wall hernias. However, recent observations of expanded (unwarranted) indications for these extensive procedures are a cause for concern. An unwarranted and incorrect surgical technique may lead to unacceptable anatomical and functional damage to the abdominal wall ("abdominal wall cripples") Laparoscopic IPOM repair, where indicated, has several advantages. It is quick to perform, easy to replicate and maintains integrity of the abdominal wall.

It is clearly evident that a continuous process of audit, course correction and innovation, both conceptual and surgical, have driven progress in surgery. The primary aim of treatment of hernia is repair of the defect in the abdominal wall, relief of symptoms and elimination of risk of bowel incarceration and strangulation. It is fair to assume that patients adapt to any loss of function of the abdominal wall over the several years with the hernia. Moreover, many of these patients are no more active in sport, profession or even daily life. It may, therefore, not be appropriate to be aggressive with attempting to restore "normal" abdominal wall function in these patients. It follows that a policy of always attempting to primarily reconstruct the midline in all patients as the primary goal of hernia repair is unnecessary 
and perhaps unwarranted. This may result in larger and more aggressive operations on the abdominal wall with increased complication rates.

\section{Compliance with ethical standards}

Conflict of interest The author(s) declare that they have no competing interests.

Ethical approval This article did not require ethical approval of any kind.

Human and animal rights This article is an invited commentary and does not contain any studies conducted on human participants and animals.

Informed consent For this type of study formal consent is not required.

\section{References}

1. Forbes S, Eskicioglu C, McLeod R, Okrainec A (2009) Metaanalysis of randomized controlled trials comparing open and laparoscopic ventral and incisional hernia repair with mesh. Br J Surg 96:851e-858

2. Olmi S, Scaini A, Cesana GC, Erba L, Croce E (2007) Laparoscopic versus open incisional hernia repair: an open randomized controlled study. Surg Endosc 21(4):555-559

3. Floros C, Davis PK (1991) Complications and long-term results following abdominoplasty: a retrospective study. Br J Plast Surg 44:190-194

4. LeBlanc KA, Booth WV (1993) Lapraroscopic repair of incisional abdominal hernias using expanded polytetraflouroethylene: preliminary findings. Surg Laparosc Endosc 3:39-41

5. Prasad P, Tantia O, Patle NM, Khanna S, Sen B (2011) Laparoscopic ventral hernia repair: a comparative study of transabdominal preperitoneal versus intraperitoneal onlay mesh repair. J Laparoendosc Adv Surg Tech A 21(6):477-83

6. Sharma A, Dey A, Khullar R, Soni V, Baijal M, Chowbey PK (2011) Laparoscopic repair of suprapubic hernias: transabdominal partial extraperitoneal (TAPE) technique. Surg Endosc 25:2147-2152

7. .Belyansky I, Zahiri HR, Park A (2016) Laparoscopic transversus abdominis release, novel minimally invasive approach to complex abdominal wall reconstruction. Surg Innov 23(2):134-141. https ://doi.org/10.1177/1553350615618290 (Epub 2015 Nov 24)
8. Askar OM (1977) Surgical anatomy of the aponeurotic expansions of the anterior abdominal wall. Ann R Coll Surg Engl 59(4):313-321

9. Ramirez OM, Ruas E, Dellon AL (1990) "Components separation" method for closure of abdominal-wall defects: an anatomic and clinical study. Plast Reconstr Surg 86:519

10. Jensen KK, Munim K, Kjaer M, Jorgensen LN (2017) Abdominal wall reconstruction for incisional hernia optimizes truncal function and quality of life: a prospective controlled study. Ann Surg 265:1235-1240

11. Kuylhee K, Mella JR, Ahmed MSI, Pieter G, Koolen L, Lin SJ (2015) Is there an association between component separation and venous thromboembolism? Analysis of the NSQIP. Plast Reconstr Surg Glob Open 3(6):e429 (published online 2015 Jul 8)

12. Criss CN, Petro CC, Krpata DM, Seafler CM, Lai N, Fiutem J, Novitsky YW, Rosen MJ (2014) Functional abdominal wall reconstruction improves core physiology and quality-of-life. Surgery 156(1):176-182

13. Arita NA, Nguyen MT, Nguyen MT et al (2015) Laparoscopic repair reduces incidence of surgical site infections for all ventral hernias. Surg Endosc 29:1769-1780

14. Sharma A, Chowbey P, Kantharia NS, Baijal M, Soni V, Khullar R (2018) Previously implanted intra-peritoneal mesh increases morbidity during re-laparoscopy: a retrospective, case-matched cohort study. Hernia 22(2):343-351

15. Ecker BL, Kuo LE, Simmons KD, Fischer JP, Morris JB, Kelz RR (2016) Laparoscopic versus open ventral repair: longitudinal outcomes and cost analysis using statewide claims data. Surg Endosc 30:906-915

16. Kokotovic F, Bisgaard T, Helgstrand F (2016) Long-term recurrence and complications associated with elective incisional hernia repair. JAMA 316:1575-1582

17. Palanivelu C, Jani KV, Senthilnathan P, Parthasarathi R, Madhankumar MV, Malladi VK (2007) Laparoscopic sutured closure with mesh reinforcement of incisional hernias. Hernia 11(3):223-228 (Epub 2007 Feb 13)

18. Clapp ML, Hicks SC, Awad SS, Liang MK (2013) Upto now there is no evidence of any superiority over standard procedure (trans-cutaneous closure of central defects. (TCCD) in laparoscopic ventral hernia repairs (LVHR). World J Surg 37:42-51

19. Suwa K, Okamoto T, Yanaga K (2016) Closure versus non-closure of fascial defects in laparoscopic ventral and incisional hernia repairs: a review of the literature. Surg Today 46:764-773

20. Hauters P, Desmet J, Gherardi D, Dewaele S, Polivache H, Malvaux $P$ (2017) Assessment of predictive factors for recurrence in laparoscopic ventral hernia using a bridging technique. Surg Endosc 31:3656-3663 